\title{
Nonlinear Distance-Based Dynamic Pricing Considering Congestion-Level Correction
}

\author{
Qixiu Cheng, Zhiyuan Liu* and Aya Selmoune \\ Jiangsu Key Laboratory of Urban ITS, Jiangsu Province Collaborative Innovation \\ Center of Modern Urban Traffic Technologies, Southeast University, Nanjing \\ 210096, China \\ *zhiyuanl@seu.edu.cn; leakeliu@163.com
}

\begin{abstract}
In order to make the congestion pricing policy more equitable and effective, and take a full consideration the time-dependent nature of traffic flow and the dynamics of users' departure time decisions, an optimal dynamic congestion pricing problem is addressed in this paper. A nonlinear distance-based toll considering the congestion-level correction is levied for road users in a charging cordon. It is assumed that both users' departure time and route choice behavior follow the dynamic user equilibrium (DUE) principle. A bilevel programming model for the nonlinear distance-based dynamic pricing which considers the congestion-level correction is formulated to determine the optimal toll rate. The upper level aims to maximize the total social benefits, while the lower level depicts user' departure time as well as route choice behavior in terms of the DUE theory. The model proposed here can be used to design the optimal toll rate for the dynamic congestion pricing.
\end{abstract}

Keywords: congestion pricing, dynamic optimal toll, nonlinear distance-based pricing, bi-level programming, dynamic user equilibrium

\section{Introduction}

Traffic congestion is a very common problem in many cities around the world, especially in the metropolitan cities. As an economic instrument for transport demand management (TDM), congestion pricing is of great significance in alleviating traffic congestion and has received more and more attention both academically and practically. Since the successful implementation of cordon-based congestion pricing in Singapore from 1975, many countries and cities (such as Norway, London, Stockholm and Milan) have implemented a road congestion pricing policy, which has achieved remarkable success in terms of easing urban traffic congestion [1-7]. A well-known theory for traffic congestion is that individual travelers imposes delays on others while they do not pay the entire marginal social cost of their personal trips [8]. Generally, the congestion pricing schemes can be divided into the first-best pricing and the second-best pricing scheme. Specifically, the first one tolls every link in the whole network [9-11], while the second one tolls only a subset of the links in the whole network [12]. Yang and Huang [13] made a comprehensive summary of mathematical formulation as well as economic theory for both two pricing manners in a static environment, and interested readers can refer to their work in these two pricing schemes.

Determining a suitable toll rate is usually regarded as one of the most important issues in the congestion pricing problem. Nevertheless, only a flat-toll method (e.g., pay per entry charging and daily licensing charging) is widely adopted by those countries or cities

Received (May 10, 2017), Review Result (April 26, 2018), Accepted (May 21, 2018)

* Corresponding Author 
which have implemented the congestion pricing policy successfully. The actual travel distance, time and congestion level of the road environment are not involved, making it inequitable and ineffective in the flat-toll method. Thus, due to the drawbacks existing in the flat-toll scheme, a nonlinear distance-based toll scheme should be adopted as an alternative to improve the fairness and the effectiveness [14-15]. However, only a distance-based toll scheme still has its limitation: travelers would deliberately choose the shorter path to reduce their toll payments in the pricing areas, regardless this route is highly congested. In order to cope with this problem, a combination toll scheme which incorporates a distance-toll as well as a time-toll function, is proposed in [16]. However, whether in the distance-toll or time-toll scheme, it contains a free-flow travel time related cost, resulting an overlap issue. Consequently, the free-flow travel time related part calculated in the time-based toll should be eliminated. Essentially, after eliminating this free-flow travel time related part of the time-based toll, the new toll scheme becomes a nonlinear distance-toll considering the correction of actual congestion-level of the road environment in the pricing cordon. Note that the delay time, which indicates the congestion level, can be calculated by the actual travel time minus the free-flow travel time.

It is necessary to incorporate the dynamic user equilibrium (DUE) theory to the congestion pricing because of the nature of time-varying traffic flow and the dynamics of users' departure time decisions [17]. As for dynamic congestion pricing, most studies [1822] are time-varying tolls without considering the travel distance, travel time, or the actual congestion level in the pricing cordon, making it inequitable and ineffective. Hence, it is essential to encapsulate the distance-toll and congestion-toll to the dynamic congestion pricing. Cheng et al., [23] made a comprehensive review of dynamic congestion pricing and highlighted that it is an emerging research need to study the dynamic congestion pricing problem. Note that the day to day dynamic congestion pricing (such as [24]) is not considered, but only a within-day dynamic toll is studied in this paper. The overall structure of this paper is organized as follows. Section 2 first introduces the notations and assumptions, and describes the research problem, including a distance-based toll considering congestion-level correction as well as a dynamic network loading (DNL) process, in this paper. Then, Section 3 proposes a DUE model in terms of the analytical method. After this, a bi-level programming model is formulated in Section 4 and a tworoute experimental study is conducted in Section 5. Finally, Section 6 draws the conclusions.

\section{Problem Description}

A strongly connected transportation network which is denoted by $G=(N, A)$, is considered in this paper. We use $N$ to denote the set of all nodes, and $A$ to denote the set of all directed links in the network. $W$ represents the set of origin-destination (OD) pairs, and $R_{w}$ is the set of all paths between an OD pair $w \in W . Q_{w}$ denotes the traffic demand between an OD pair $w \in W$. Other notations and corresponded explanations are listed in Table 1.

Table 1. A Summary of Notations used in this Paper

\begin{tabular}{ll}
\hline Notations & Explanations \\
\hline$N$ & Set of all nodes \\
$n$ & $n \in N$ \\
$A$ & Set of all links \\
$a$ & $a \in A$ \\
$\left\{a_{1}, a_{2}, \cdots, a_{m(p)}\right\}=p$ & Links on path $p$ \\
$m(p)$ & Number of links on path $p$
\end{tabular}




\begin{tabular}{ll}
\hline Notations & Explanations \\
\hline$P$ & The set of all paths \\
$P_{w}$ & The set of all paths connecting an OD pair $w \in W$ \\
$p$ & $p \in P$ \\
$Q_{w}$ & The traffic demand of an OD pair $w \in W$ \\
$t_{0}$ & Free-flow travel time on path $p$ \\
$T_{a}$ & Time traveled on link $a$ \\
$T_{p}$ & Time traveled on path $p$ \\
$T_{A}$ & Desired arrival time \\
$k$ & Departure time index \\
$x_{a}^{p}(t)$ & Volume on link $a$ of path $p$ when the departure time is $t$ \\
$x_{a}(t)$ & Volume on link $a$ when the departure time is $t$ \\
$h_{p}(t)$ & Flow entering path $p$ when the departure time is $t$ \\
$h_{p}^{*}(t)$ & Equilibrium flow entering path $p$ when the departure time is $t$ \\
$g_{a}^{p}(t)$ & Exit flow on link $a$ along path $p$ when the departure time is $t$ \\
$y_{a}(t)$ & The toll for link $a$ at time $t$ \\
$y_{p}(t)$ & The toll for path $p$ at time $t, y_{p}(t)=\sum_{\mathrm{i}=1}^{m(p)} y_{\left(a_{i}\right)}(t)$ \\
$\xi_{a}^{p}$ & The exit time from link $a$ of path $p$ \\
$\theta_{p}$ & Tolled effective delay operator of path $p$ \\
$\psi_{p}$ & Effective delay operator of path $p$ \\
\hline
\end{tabular}

\subsection{Nonlinear Distance-based Toll Considering Congestion-level Correction}

We can formulate the nonlinear distance-toll function $\phi(\eta, t)$ as a piecewise linear function in term of the travel distance $\eta$ at time $t$. Assuming that the minimal and maximal length of all paths in the corresponding cordons are $\eta_{0}$ and $\eta_{K}$, then we can divide the travel distance into $K$ equal intervals and the distance-toll function of each interval can be expressed by the two endpoints. This method is first introduced in [14] and consummated by adding binary variables so as to reduce the number of constraints in [16]. We also use this method to describe the nonlinear distance-toll function in this paper.

As discussed above, each distance-toll $\phi_{k}$ in $\Phi=\left(\phi_{0}, \phi_{1}, \cdots, \phi_{k}, \cdots, \phi_{K}\right)^{T}$ can only be the discrete values from $\phi^{\min }$ to $\phi^{\max }$ with the increment of $\Delta_{\phi}$, scilicet:

$$
\phi \in \Pi_{\phi}=\left\{\phi^{\min }, \phi^{\min }+\Delta_{\phi}, \cdots, \phi^{\min }+k \Delta_{\phi}, \cdots, \phi^{\min }+\left\lfloor\frac{\phi^{\max }-\phi^{\min }}{\Delta_{\phi}}\right\rfloor \Delta_{\phi}\right\}, k=1, \cdots, K
$$

The cardinality of set $\Pi_{\phi}$ can be measured as:

$$
\left|\Pi_{\phi}\right|=\left\lfloor\frac{\phi^{\max }-\phi^{\min }}{\Delta_{\phi}}\right\rfloor+1
$$

where the symbol of " \lfloor\rfloor " is used to calculate the smallest integer that is greater than or equal to the number itself.

So we can use some binary variables, which are denoted by $\tilde{z}_{k j}$, to obtain each distance-toll $\phi_{k}$ : 


$$
\begin{aligned}
& \phi_{k}=\sum_{j=0}^{\left|\Pi_{\phi}\right|-1}\left(\phi^{\min }+j \Delta_{\phi}\right) \tilde{z}_{k j}, \quad j=0,1, \cdots,\left|\Pi_{\phi}\right|-1 ; k=1,2, \cdots, K \\
& \sum_{j=0}^{\left|\Pi_{\phi}\right|-1} \tilde{z}_{k j}=1, \quad j=0,1, \cdots,\left|\Pi_{\phi}\right|-1 ; k=1,2, \cdots, K \\
& \tilde{z}_{k j} \in\{0,1\}, \quad j=0,1, \cdots,\left|\Pi_{\phi}\right|-1 ; \quad k=1,2, \cdots, K
\end{aligned}
$$

It is easy to find that the total number of binary variables is $\left|\Pi_{\phi}\right| \times K$. However, for the sake of improving the computational efficiency and reducing the total number of binary variables as well as constraints, the distance-toll $\phi_{k}$ can be alternatively calculated by the following formulation:

$$
\begin{aligned}
\phi_{k} & =\phi^{\min }+2^{0} z_{k 0} \Delta_{\phi}+2^{1} z_{k 1} \Delta_{\phi}+2^{2} z_{k 2} \Delta_{\phi}+\cdots+2^{j} z_{k j} \Delta_{\phi}+\cdots+2^{J} z_{k J} \Delta_{\phi} \\
& =\phi^{\min }+\sum_{j=0}^{J} 2^{j} z_{k j} \Delta_{\phi} \\
z_{k j} & \in\{0,1\}, \quad j=0,1, \cdots, J ; k=1,2, \cdots, K \\
J & =\left\lfloor\log _{2}\left(\left|\Pi_{\phi}\right|-1\right)\right\rfloor
\end{aligned}
$$

It is obvious that the essential number of binary variables is $(J+1) \times K$. We use the column vector $\mathbf{z}=\left(z_{k j}, 1 \leq k \leq K, 0 \leq j \leq J\right)^{T}$ to denote the feasible set of binary variables, then the distance-based toll design problem becomes determining the optimal $\mathbf{z}$ at time $t$.

Compared with the time-based toll charge scheme proposed by Liu et al., [16], the congestion-based toll is related with the travel time-consuming in congestion, which is calculated by the function with time in excess of free-flow travel time. The time spent in congestion is also called delay time. Therefore, similar with the time-toll function, the congestion-toll function at time ${ }^{t}$ is represented by:

$$
\varphi(\Delta t, t)=\beta \cdot \Delta t
$$

where $\varphi(\Delta t, t)$ is assumed to be proportional to the delay time $\Delta t$ in the cordon at time $t$ and $\beta$ is a positive pricing rate with the time spent in the congestion.

Actually with the occurrence of congestion in the cordon, the delay time $\Delta t$ of path $p$ can be calculated as follows:

$$
\Delta t=T_{p}-t_{0}=\xi_{a_{m(p)}}^{p}-t-t_{0}
$$

Hence, the congestion-based toll design problem becomes determining the optimal $\beta$ in the cordon.

Based on the distance-toll and congestion-toll proposed above, we can formulate a new toll function $y$, which is a weighted summation of the distance-toll function $\phi$ and congestion-toll function $\varphi$ namely:

$$
y_{p}(\mathbf{z}, \beta, t)=\lambda_{1} \phi(\mathbf{z}, t)+\lambda_{2} \varphi(\beta, t)
$$

where $\lambda_{1}$ and $\lambda_{2}$ are the weights of distance-toll and congestion-toll. Eq. (11) is actually a new distance-toll function considering congestion-level correction, and it is uniquely determined by the set of binary variables $\mathbf{z}$, congestion-toll rate $\beta$ and the departure time $t$. 


\subsection{Dynamic Network Loading}

We assume that the analysis time interval is $\left[t_{0}, t_{f}\right]$, where $t_{0}<t_{f}$. For each link on path $p=\left\{a_{1}, a_{2}, \cdots, a_{m(p)}\right\} \in P$, we can get the state equations of each link as follows $[17,25]$ :

$$
\begin{array}{ll}
\frac{d x_{a_{1}}^{p}}{d t}=h_{p}(t)-g_{a_{1}}^{p}(t) & \forall p \in P \\
\frac{d x_{a_{n}}^{p}}{d t}=g_{a_{i-1}}^{p}(t)-g_{a_{i}}^{p}(t) & \forall p \in P, i \in[2, m(p)]
\end{array}
$$

where $h_{p}(t)$ denotes the enter flow on path $p$ at time $t, g_{a_{i}}^{p}(t)$ denotes the exit flow on link $a_{i}$ of path $p$ at time $t, x_{a_{i}}^{p}(t)$ denotes the volume on link $a_{i}$ of path $p$ at time $t$.

The volume on link $a_{i}$ at time $t$ can be expressed as:

$$
x_{a_{i}}(t)=\sum_{p \in P} \delta_{a_{i} p} x_{a_{i}}^{p}(t) \quad \forall p \in P
$$

where $\delta_{a_{i} p}$ is an indicator variable, $\delta_{a_{i} p}=1$ if link $a_{i}$ is on path $p$, and $\delta_{a_{i} p}=0$ otherwise.

In order to obtain the flow propagation function, we should get the exit time from each link $a_{i}$ of path $p$. The exit time function $\xi_{a_{1}}^{p}$ can be defined as:

$$
\begin{aligned}
& \xi_{a_{1}}^{p}=t+T_{a_{1}}\left[x_{a_{1}}(t)\right] \quad \forall p \in P \\
& \xi_{a_{i}}^{p}=\xi_{a_{i-1}}^{p}(t)+T_{a_{i}}\left[x_{a_{i}}\left(\xi_{a_{i-1}}^{p}(t)\right)\right] \quad \forall p \in P, i \in[2, m(p)]
\end{aligned}
$$

where $T_{a_{i}}\left[x_{a_{i}}(t)\right]$ denotes the time required to travel on link $a_{i}$.

Differentiating Eqs. (15) and (16) with respect to the time, and we can obtain the following functions:

$$
\begin{aligned}
\frac{d \xi_{a_{1}}^{p}}{d t} & =1+T_{a_{1}}^{\prime}\left[x_{a_{1}}(t)\right] \cdot \frac{d x_{a_{1}}(t)}{d t} \quad \forall p \in P \\
\frac{d \xi_{a_{i}}^{p}}{d t} & =\frac{d \xi_{a_{i-1}}^{p}(t)}{d t}+T_{a_{i}}^{\prime}\left[x_{a_{i}}\left(\xi_{a_{i-1}}^{p}(t)\right)\right] \cdot \frac{d x_{a_{i}}\left[\xi_{a_{i-1}}^{p}(t)\right]}{d \xi_{a_{i-1}}^{p}(t)} \cdot \frac{d \xi_{a_{i-1}}^{p}(t)}{d t} \\
& =\left[1+T_{a_{i}}^{\prime}\left[x_{a_{i}}\left(\xi_{a_{i-1}}^{p}(t)\right)\right] \cdot \frac{d x_{a_{i}}\left[\xi_{a_{i-1}}^{p}(t)\right]}{d \xi_{a_{i-1}}^{p}(t)}\right] \cdot \frac{d \xi_{a_{i-1}}^{p}(t)}{d t} \\
& \forall p \in P, i \in[2, m(p)]
\end{aligned}
$$

According to Friesz et al., [25], the enter flow function can be expressed as:

$$
h_{p}(t)=g_{a_{i}}^{p}\left[\xi_{a_{i}}^{p}(t)\right] \cdot \frac{d \xi_{a_{i}}^{p}(t)}{d t}
$$

By substituting Eqs. (17) and (18) into (19), we can obtain:

$$
\begin{aligned}
& h_{p}(t)=g_{a_{1}}^{p}\left(t+T_{a_{1}}\left[x_{a_{1}}(t)\right] \cdot\left(1+T_{a_{1}}^{\prime}\left[x_{a_{1}}(t)\right] \cdot \dot{x}_{a_{1}}\right)\right. \\
& g_{a_{i-1}}^{p}=g_{a_{i}}^{p}\left(t+T_{a_{i}}\left[x_{a_{i}}(t)\right] \cdot\left(1+T_{a_{i}}^{\prime}\left[x_{a_{i}}(t)\right] \cdot \dot{x}_{a_{i}}(t)\right) \quad \forall p \in P, i \in[2, m(p)]\right.
\end{aligned}
$$


where the "' superscript represents the differentiation, while the dot ." is the time derivation.

We can obtain the total travel time for path $p$ :

$$
T_{p}(t, h(t))=\xi_{a_{1}}^{p}-t+\sum_{i=2}^{m(p)}\left[\xi_{a_{i}}^{p}(t)-\xi_{a_{i-1}}^{p}(t)\right]=\xi_{a_{m(p)}}^{p}(t)-t \quad \forall p \in P
$$

According to Chung et al., [17], the effective delay contains an arrival penalty operator which can be expressed as $F(\cdot)$. When the desired arrival time $T_{A}$ is not equal to the actual arrival time $t+T_{p}(t, h(t))$, we can use the penalty operator to express the effective delay operator $\psi_{p}$ :

$$
\psi_{p}(t, h(t))=T_{p}(t, h(t))+F\left(t+T_{p}(t, h(t))-T_{A}\right) \quad \forall p \in P
$$

By substituting Eq. (22) into (23), then we can compute the effective delay operator as a function of path exit time $\xi_{a_{m(p)}^{p}}^{p}$ :

$$
\psi_{p}(t, h(t))=\xi_{a_{m(p)}}^{p}-t+F\left[\xi_{a_{(p)}}^{p}(t)-T_{A}\right]
$$

\section{Dynamic User Equilibrium}

We can solve the DUE problem based on the DNL presented in Section 2.2. In addition to the arrival penalty operator, the effective delay operator also includes a toll operator after the implementation of congestion pricing. Thus, for pricing cordon areas, the tolled effective delay operator can be expressed as:

$$
\theta_{p}(t, h(t), y(t))=T_{p}+F\left(t+T_{p}-T_{A}\right)+y_{p}(t) \quad \forall p \in P
$$

where $y_{p}(t)$ denotes the toll for path $p$ at time $t$.

By substituting Eq. (23) into (25), we can obtain:

$$
\begin{aligned}
& \theta_{p}(t, h(t), y(t))=\psi_{p}(t, h(t))+y_{p}(t) \\
& y_{p}(t)=\sum_{i=1}^{m(p)} y_{a_{i}}(t)=y_{a_{1}}(t)+\sum_{i=2}^{m(p)} y_{a_{i}}\left(\xi_{a_{i-1}}^{p}(t)\right)
\end{aligned}
$$

For each OD pair, at dynamic user equilibrium, the instantaneous travel impedances for all chosen routes are the same and also no more than the impedance which would be potentially experienced by a single vehicle on any unused route at any decision point of any time. Based on this definition, we can obtain the mathematical representation of DUE condition which can be formulated as:

$$
\begin{aligned}
& \sum_{p \in P} \int_{t_{0}}^{t_{f}} \theta_{p}\left(t, h^{*}(t), y(t)\right) \cdot\left(h(t)-h^{*}(t)\right) d t \geq 0, \quad h^{*}(t) \in \Omega, \forall h(t) \in \Omega \\
& \Omega=\left\{\sum_{p \in P_{w}} \int_{t_{0}}^{t_{f}} h_{p}(t) d t=Q_{w}, h_{p}(t) \geq 0, w \in W\right\}
\end{aligned}
$$

It should be noted that the proof of equivalence of DUE condition and the variational inequality (28) can be found in Friesz et al., [26], which is not described in detail here.

\section{Bi-level Programming Model Formulation}

Optimal dynamic congestion pricing problem can be formulated as a bi-level programming model, with the upper-level of maximizing the total social benefit (TSB), and the lower-level of deterministic DUE, which can be expressed as: 


$$
\begin{gathered}
\max \operatorname{TSB}=\sum_{p \in P_{w}} \sum_{w \in W} \int_{t_{0}}^{t_{f}} \int_{0}^{q_{w}} D^{-1}(x, t) d x d t-\sum_{p \in P_{w}} \sum_{w \in W} \int_{t_{0}}^{t_{f}} h_{p}^{*}(t) \psi_{p}\left(t, h^{*}(t)\right) d t \\
\quad+\sum_{p \in P_{w}} \sum_{w \in W} \int_{t_{0}}^{t_{f}} \frac{y_{p}(\mathbf{z}, \beta, t)}{\alpha} d t \\
\text { s.t. } \quad \sum_{p \in P_{w}} \sum_{w \in W} \int_{t_{0}}^{t_{f}} \theta_{p}\left(t, h^{*}(t), y(t)\right) \cdot\left(h(t)-h^{*}(t)\right) d t \geq 0, \\
\quad h^{*}(t) \in \Omega, \forall h(t) \in \Omega \\
\quad \Omega=\left\{\sum_{p \in P_{w}} \int_{t_{0}}^{t_{f}} h_{p}(t) d t=Q_{w}, h_{p}(t) \geq 0, w \in W\right\}
\end{gathered}
$$

\section{Two-route Case Study}

In this section, we provide a simple case study with a two-route network to illustrate the effectiveness of the proposed methodology in this paper. As shown in Figure 1, this network has one OD pair from node 1 to node 3, with a total demand of 10 units. Link 2 is a tolled link, while links 1 and 3 are un-tolled links. The attributes of this network are summarized in Table 2.

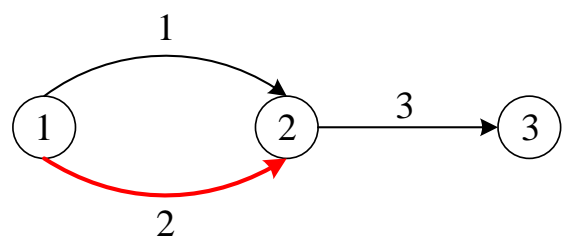

Figure 1. Network Representation with Two-Route Alternatives

Table 2. Network Attributes

\begin{tabular}{cccccc}
\hline Index & Link & From & To & Length & Unit Cost \\
\hline 1 & $a_{1}$ & 1 & 2 & 1 & $1+2 x_{1}$ \\
2 & $a_{2}$ & 1 & 2 & 1 & $1+x_{2}+y$ \\
3 & $a_{3}$ & 2 & 3 & 1 & $1+x_{3}$ \\
\hline
\end{tabular}

According to the computational results of this two-route experiment, we can obtain that the optimal toll for link 2 is 2.7 , and link flows are 4.23, 5.77 and 10 for links $1,2,3$ respectively. This is the first step to validate the methodology proposed in this paper. In future research, we will test it on a much larger network and to incorporate more detailed scenarios with the dynamic pricing problem.

\section{Conclusions}

A bi-level programming model for the dynamic congestion pricing problem is developed. The upper level problem aims to maximize the TSB, while the lower level is a DUE problem. The nonlinear distance-based toll function as well as the congestion-based toll function are encapsulated to the dynamic congestion pricing. Travelers' departure time and route choice behavior are considered in the DUE formulation. This proposed nonlinear distance-based dynamic optimal toll taking into consideration actual congestion level adequately considers the equity and efficiency of the network. The model proposed in this paper can be used to design the optimal toll rate for the dynamic congestion pricing. 


\section{Acknowledgments}

This study is supported by the Youth Projects (No. 71501038), General Projects (No. 71771050), Key Projects (No. 51638004) of the National Natural Science Foundation of China, and the Natural Science Foundation of Jiangsu Province in China (BK20150603).

\section{References}

[1] S. Y. Phang and R. S. Toh, "From manual to electronic road congestion pricing: The Singapore experience and experiment", Transportation Research Part E, vol. 33, no. 2, pp. 97-106.

[2] M. Li, "Estimating congestion toll by using traffic count data-Singapore's area licensing scheme", Transportation Research Part E, vol. 35, no. 1, (1999), pp. 1-10.

[3] F. T. Seik, "An advanced demand management instrument in urban transport: electronic road pricing in Singapore", Cities, vol. 17, no. 1, (2000), pp. 33-45.

[4] T. Langmyhr, "Learning from road pricing experience: introducing a second-generation road pricing system", Planning Theory \& Practice, vol. 2, no. 1, (2001), pp. 67-80.

[5] G. Santos and G. Fraser, "Road pricing: lessons from London", Economic Policy, vol. 21, no. 46, (2006), pp. 264-310.

[6] J. Eliasson, "A cost-benefit analysis of the Stockholm congestion charging system", Transportation Research Part A, vol. 43, no. 4, (2009), pp. 468-480.

[7] L. Rotaris, R. Danielis and E. Marcucci, "The urban road pricing scheme to curb pollution in Milan, Italy: Description, impacts and preliminary cost-benefit analysis assessment”, Transportation Research Part A, vol. 44, no. 5, (2010), pp. 359-375.

[8] A. De Palma and R. Lindsey, "Traffic congestion pricing methodologies and technologies", Transportation Research Part C, vol. 19, no. 6, (2011), pp. 1377-1399.

[9] R. Arnott and K. Small, "The economics of traffic congestion”, American scientist, (1994), pp. 446-455.

[10] R. B. Dial, "Minimal-revenue congestion pricing part I: A fast algorithm for the single-origin case", Transportation Research Part B, vol. 33, no. 3, (1999), pp. 189-202.

[11] R. B. Dial, "Minimal-revenue congestion pricing part II: An efficient algorithm for the general case", Transportation Research Part B, vol. 34, no. 8, (2000), pp. 645-665.

[12] S. Lawphongpanich and D. W. Hearn, "An MPEC approach to second-best toll pricing", Mathematical Programming, vol. 101, no. 1, (2004), pp. 33-55.

[13] H. Yang and H. J. Huang, "Mathematical and economic theory of road pricing", Elsevier, Oxford, (2005).

[14] Q. Meng, Z. Liu and S. Wang, "Optimal distance tolls under congestion pricing and continuously distributed value of time”, Transportation Research Part E, vol. 48, no. 5, (2012), pp. 937-957.

[15] X. Sun, D. Huang and Q. Cheng, "Optimal Nonlinear Distance Toll for Cordon-Based Congestion Pricing Considering Equity Issue”, Journal of Harbin Institute of Technology, vol. 23, no. 6, (2016), pp. 73-79.

[16] Z. Liu, S. Wang and Q. Meng, "Optimal joint distance and time toll for cordon-based congestion pricing", Transportation Research Part B, vol. 69, (2014), pp. 81-97.

[17] B. D. Chung, T. Yao and T. L. Friesz, "Dynamic congestion pricing with demand uncertainty: A robust optimization approach", Transportation Research Part B, vol. 46, no. 10, (2012), pp. 1504-1518.

[18] H. Yang and H. J. Huang, "Analysis of the time-varying pricing of a bottleneck with elastic demand using optimal control theory", Transportation Research Part B, vol. 31, no. 6, (1997), pp. 425-440.

[19] H. Yang and Q. Meng, "Departure time, route choice and congestion toll in a queuing network with elastic demand", Transportation Research Part B, vol. 32, no. 4, (1998), pp. 247-260.

[20] N. Zheng, R. A. Waraich and K. W. Axhausen, "A dynamic cordon pricing scheme combining the Macroscopic Fundamental Diagram and an agent-based traffic model", Transportation Research Part A, vol. 46, no. 8, (2012), pp. 1291-1303.

[21] K. Zhang, H. S. Mahmassani and C. C. Lu, "Dynamic pricing, heterogeneous users and perception error: Probit-based bi-criterion dynamic stochastic user equilibrium assignment", Transportation Research Part C, vol. 27, (2013), pp. 189-204.

[22] K. Jang, K. Chung and H. Yeo, "A dynamic pricing strategy for high occupancy toll lanes", Transportation Research Part A, vol. 67, (2014), pp. 69-80.

[23] Q. Cheng, Z. Liu and F. Liu, "Urban dynamic congestion pricing: an overview and emerging research needs", International Journal of Urban Sciences, (2016), pp. 1-16.

[24] Z. Liu, S. Wang and B. Zhou, "Robust Optimization of Distance-based Tolls in a Network Considering Stochastic Day to Day Dynamics", Transportation Research Part C, vol. 79, (2017), pp. 58-72.

[25] T. L. Friesz, D. Bernstein and Z. Suo, "Dynamic network user equilibrium with state-dependent time lags", Networks and Spatial Economics, vol. 1, no. 3, (2001), pp. 319-347.

[26] T. L. Friesz, D. Bernstein and T. E. Smith, "A variational inequality formulation of the dynamic network user equilibrium problem”, Operations Research, vol. 41, no. 1, (1993), pp. 179-191. 